\title{
Positive Correlation between Serum Osteocalcin and Testosterone in Male Hyperthyroidism Patients with High Bone Turnover
}

Authors

Affiliation

\section{N. Zhong*, B. Xu*, R. Cui, M. Xu, J. Su, Z. Zhang, Y. Liu, L. Li, C. Sheng, H. Sheng, S. Qu}

Department of Endocrinology and Metabolism, Shanghai Tenth People's Hospital, Tongji University School of Medicine, China
Key words

osteocalcin

- testosterone

- hyperthyroidism

received 28.02.2016

first decision 29.04.2016

accepted $\quad 03.05 .2016$

Bibliography

DoI http://dx.doi.org/

10.1055/s-0042-107944

Published online:

May 24, 2016

Exp Clin Endocrinol Diabetes

2016; 124: 452-456

(c) J. A. Barth Verlag in

Georg Thieme Verlag KG

Stuttgart · New York

ISSN 0947-7349

Correspondence

Prof. $\boldsymbol{H}$. Sheng

Department of Endocrinology

and Metabolism

Shanghai Tenth People's

Hospital

Tongji University School of

Medicine

China

Tel.: + 86/133/86052 209

shenghui@tongji.edu.cn

\section{Abstract \\ V}

Purpose: Animal studies suggested that there is an independent bone-osteocalcin-gonadal axis, except of the hypothalamic-pituitary-gonadal axis. Based on this hypothesis, the higher osteocalcin during the high bone turnover should be followed by higher testosterone formation. Yet such clinical evidence is limited. The patients with uncontrolled hyperthyroidism are proper model with high bone turnover. If this hypothesis is true, there should be high testosterone level in patients with uncontrolled hyperthyroidism. Therefore, Graves' disease patients were recruited to study the correlation between osteocalcin and testosterone.

Materials and Methods: 50 male hyperthyroidism patients with Graves' disease and 50 health persons matched by age and gender were enrolled in our cross-section study. Serum markers for thyroid hormone, sex hormone and bone metabolic markers including free tri-

\section{Introduction}

$\nabla$

It is well known that testosterone in male is mainly controlled by hypothalamus-pituitarytestis axis, in which circulating levels of luteinizing hormone (LH), a pituitary hormone, acts as an primary upstream regulators of sex steroid [1-3]. However, a round-new finding in animal studies showed that osteocalcin (OC), synthesized and secreted by osteoblasts, could regulate male reproductive functions by promoting testosterone biosynthesis in Leydig cells [4-6], which suggests there is a pancreas-bone-testis axis. Oury's study found that osteocalcin-null (Osteocalcin-/-) male mice's testosterone was lower than wild type(WT) mice. To further iden-

* Dr. Ni Zhong and Dr. Bei Xu contribute equally to this paper iodothyronine (FT3), free thyroxine (FT4), thyroid-stimulating hormone (TSH), testosterone, luteinizing hormone (LH), follicle-stimulating hormone (FSH) and osteocalcin (OC), C-terminal telopeptide fragments of type I collagen (CTX) were examined. The demographic parameters such as duration of disease were also collected. All data was analyzed by SPSS 20.0.

Results: High testosterone and osteocalcin level was observed in the hyperthyroidism patients ( $\mathrm{T} 36.35 \pm 10.72 \mathrm{nmol} / \mathrm{l}$ and OC $46.79 \pm 26.83 \mathrm{ng} /$ $\mathrm{ml})$. In simple Pearson correlation, testosterone was positively associated with OC $(r=0.486$, $\mathrm{P}<0.001$ ), and this positive relation still existed after adjusted for age, BMI, smoking, drinking, duration of disease, FT3, FT4, LH, FSH, CTX in multi-linear regression analysis (See Model 1-4). Conclusion: In male hyperthyroidism patients, osteocalcin was positively correlated with serum testosterone, which indirectly supports the hypothesis that serum osteocalcin participates in the regulation of sex hormone.

tify whether osteocalcin has positive influence to testosterone, Oury et al. injected osteocalcin to Osteocalcin-/- mice, and then found that the sperm count, testis, epididymal and seminal vesicle weights normalized, and circulating testosterone levels was higher after Osteocalcin-/- mice injected with OC, which suggests osteocalcin can promote testosterone production [4]. Additionally, accumulating evidence in recent years has come up with that skeleton is an endocrine organ [7-9]. It is said that osteocalcin not only regulate bone mineral metabolism, but also participate in lipid metabolism and promote glucose homeostasis by acting in various tissues, such as muscle, liver and fat playing a role in metabolic syndrome $[8,9]$. What's more, sex steroid hormones play a crucial role during the bone growth spurts of puberty, and for maintenance of bone mass. In view of feedback theory, bone will in turn act on 
gonads. An epidemiological study including 1338 men found that osteocalcin is positively associated with testosterone in general population [10]. Another clinical study also demonstrated that total osteocalcin in serum could predict testosterone level in male type 2 diabetes mellitus [11]. However, more clinical evidence is needed to support this hypothesis.

Osteocalcin, usually employed as bone-forming parameter during bone turnover, was synthesized and secreted by osteoblasts, stored in the bone matrix and can be released into blood. In condition of high bone turnover such as postmenopausal women and hyperthyroidism patients, serum osteocalcin will elevate. Hyperthyroidism is the most common clinical model with high bone turnover. If there is a pancreas-bone-testis axis, there must be a positive relationship between osteocalcin and testosterone. Therefore, a cross section study was performed in male patients with hyperthyroidism to identify whether circulating levels of total testosterone is related with osteocalcin. And then the mechanism how osteocalcin promote testosterone biosynthesis or regulate male reproductive functions will be further explored.

\section{Materials and Methods}

$\nabla$

\section{Subjects}

This study was conducted in the Department of Endocrinology and Metabolism, Shanghai Tenth People's Hospital, Tongji University School of Medicine from July 2014 to July 2015. Fifty male patients diagnosed as Graves' disease (Age: $48.96 \pm 5.86$ years), were recruited from the inpatients department of Shanghai Tenth People's Hospital. Fifty non-hyperthyroidism male participants matched by age and gender, were taken as control.

\section{Inclusion criteria and exclusion criteria}

Inclusion criteria: 1) male patients first diagnosed as Graves' disease,FT3 $\geq-6.5 \mathrm{pmol} / \mathrm{l}$ (normal range $3.5-6.5 \mathrm{pmol} / \mathrm{l}), \mathrm{FT} 4 \geq 31 \mathrm{pmol} / \mathrm{l}$ (normal range, $10.2-31 \mathrm{pmol} / \mathrm{l}$ ), $\mathrm{TSH} \leq 0.35 \mathrm{mU} / \mathrm{L}$ (normal range $0.35-5.5 \mathrm{mU} / \mathrm{L}$ ). Exclusion criteria: 1 ) the patients with isotope therapy; 2 ) the patients taking drugs affecting bone metabolism such as anti-osteoporosis agents: bisphosphonates, SERMs, calcitonin, androgen, strontium and parathyroid hormone; 3) the patients with primary or secondary hypogonadism; 4) the patients with diseases affecting bone metabolism, such as recent fracture, chronic liver or renal insufficiency and hyperparathyrodism. Finally, 50 male patients were enrolled in this study. Human investigation was performed according to the principles of the Declaration of Helsinki. All subjects agreed to participate in the study with written informed consent and the protocol was approved by the Ethics Committee of Shanghai Tenth People's Hospital, Tongji University School of Medicine.

\section{The demographic parameters}

Age, duration of disease, Weight, height, alcohol use and smoking history was collected. And body mass index (BMI) was calculated by dividing the weight by height squared $\left(\mathrm{kg} / \mathrm{m}^{2}\right)$. Current smoking was defined as having smoked 100 cigarettes in one's lifetime and currently smoking cigarettes [12]. Current drinking was defined as alcohol intake more than once per month during the past 12 months [12].

\section{Serum biomarkers}

Fasting blood samples was collected after overnight (early in the morning after an overnight fast, with the last meal generally
$10 \mathrm{~h}$ before the blood draw). Thyroid hormone, including FT3, FT4 and TSH concentration were examined with standard biochemical methods. Bone metabolic markers including C-terminal telopeptide fragments of type I collagen (CTX) and parathyroid hormone (PTH), as well as sex hormone, including testosterone, luteinizing hormone ( $\mathrm{LH})$ and follicle-stimulating hormone (FSH) were tested with electrochemical luminescence (ELISA) method by Roche cobase601 immune analyzer. Serum total OC was measured using its more stable breakdown fragment form, the large $\mathrm{N}$-terminal-mid-region fragment ( $\mathrm{N}-\mathrm{Mid}$ fragment; amino acids 1-43), for intact osteocalcin is unstable. The stable N-MID-fragment was measured using an N-MID Osteocalcin ELISA kit (Elecsys, Roche diagnostic Ltd., Switzerland). Testosterone in blood was tested by The Elecsys Testosterone II assay based on a competitive test principle using a high affinity monoclonal antibody (sheep) specifically directed against testosterone. (http://www.roche-diagnostics.cz).

\section{Statistical analysis}

The normality of the distribution was tested by the KolmogorovSmirnov statistical. Data were presented as mean \pm standard deviation (SD). Comparisons of baseline demographic and biochemical parameters between groups were done by paired Student's t-test. The correlation between testosterone and osteocalcin or other parameters in the male hyperthyroidism patients was analyzed with Pearson correlation or multiple linear regression analysis. All analysis was calculated by Statistical Product and Service Solutions 20.0 (SPSS 20.0). Statistical significance was set at $\mathrm{P}<0.05$.

\section{Results}

$\nabla$

\section{Comparisons of basic parameters between the}

\section{hyperthyroidism and control group}

The baseline anthropometric and biochemical characteristics of hyperthyroidism and control group were presented in $\odot$ Table 1 . In these male hyperthyroidism patients, the population of currently smoking account for $56 \%(n=28)$ and currently drinking account for $22 \%(n=11)$. Corresponding ratio among the control group is separately $54 \%(n=27)$ and $26 \%(n=13)$. The hyperthyroidism group had significantly higher thyroid hormone $(\mathrm{P}<0.001)$ and lower TSH $(\mathrm{P}<0.001)$ than the control group, which was consistent with the diagnostic criteria of hyperthyroidism patients. The other adjusted variable for testosterone like PTH, VitD, LH and FSH had no significant difference between the 2 groups. The average BMI of this population was smaller (BMI: $21.56 \pm 2.75, \mathrm{P}=0.001)$ than the control. In addition, total cholesterol (TC $3.44 \pm 0.94 \mathrm{mmol} / \mathrm{l}, \mathrm{P}<0.001$ ) were lower than the control. Whereas the bone metabolism markers like CTX $(2.01 \pm 2.31 \mathrm{ng} / \mathrm{ml}$ vs. $0.42 \pm 0.27 \mathrm{ng} / \mathrm{ml} ; \mathrm{P}<0.001)$ and osteocalcin levels $(46.79 \pm 26.83 \mathrm{ng} / \mathrm{ml}$ vs. $13.39 \pm 5.23 \mathrm{ng} / \mathrm{ml} ; \mathrm{P}<0.001)$ as well as the male fertility marker testosterone (36.35 \pm $10.72 \mathrm{nmol} / \mathrm{l}$ vs. $13.63 \pm 5.79 \mathrm{nmol} / \mathrm{l} ; \mathrm{P}<0.001$ ) were significantly higher in the hyperthyroidism group ( $\bullet$ Table $\mathbf{1}$ ).

\section{Association between testosterone level and other parameters in male hyperthyroidism}

In the simple Pearson correlation analysis, the correlation between testosterone and respective parameter showed that testosterone level was negatively related with age $(r=-0.354$, $\mathrm{P}=0.012)$, and positively related with $\mathrm{OC}(\mathrm{r}=0.486, \mathrm{P}<0.001)$ 
Table 1 Comparison of the baseline between the hyperthyroidism group and the control group.

\begin{tabular}{|c|c|c|c|}
\hline Patients & $\begin{array}{l}\text { hyperthyroid- } \\
\text { ism group }\end{array}$ & $\begin{array}{l}\text { control } \\
\text { group }\end{array}$ & $\mathbf{P}$ \\
\hline Number & 50 & 50 & \\
\hline Age (years old) & $48.96 \pm 5.86$ & $48.96 \pm 5.86$ & \\
\hline Current smoking (n/\%) & $28(56 \%)$ & 27 (54\%) & \\
\hline Current drinking (n/\%) & $11(22 \%)$ & $13(26 \%)$ & \\
\hline Duration (years) & $6.06 \pm 9.23$ & 0 & \\
\hline $\operatorname{BMI}\left(\mathrm{kg} / \mathrm{m}^{2}\right)$ & $21.56 \pm 2.75$ & $26.25 \pm 6.46$ & $0.001^{*}$ \\
\hline $\mathrm{TC}(\mathrm{mmol} / \mathrm{l})$ & $3.44 \pm 0.94$ & $4.93 \pm 1.35$ & $<0.001^{*}$ \\
\hline Osteocalcin (ng/ml) & $46.79 \pm 26.83$ & $13.39 \pm 5.23$ & $<0.001^{*}$ \\
\hline CTX (ng/ml) & $2.01 \pm 2.31$ & $0.42 \pm 0.27$ & $<0.001^{*}$ \\
\hline $25(\mathrm{OH}) \mathrm{VitD}(\mathrm{mmol} / \mathrm{l})$ & $57.3 \pm 20.32$ & $56.46 \pm 19.64$ & $>0.05$ \\
\hline PTH (pmol/l) & $4.22 \pm 2.67$ & $4.06 \pm 2.64$ & $>0.05$ \\
\hline Testosterone (nmol/l) & $36.35 \pm 10.72$ & $13.62 \pm 5.79$ & $<0.001^{*}$ \\
\hline $\mathrm{FSH}(\mathrm{IU} / \mathrm{L})$ & $9.63 \pm 7.12$ & $9.09 \pm 8.05$ & $>0.05$ \\
\hline LH (ng/ml) & $10.65 \pm 5.57$ & $7.89 \pm 4.92$ & $>0.05$ \\
\hline FT3 (pmol/l) & $15.67 \pm 8.42$ & $4.88 \pm 0.59$ & $<0.001^{*}$ \\
\hline FT4 (pmol/l) & $43.07 \pm 28.39$ & $16.84 \pm 3.50$ & $<0.001^{*}$ \\
\hline TSH (Mu/l) & $0.40 \pm 1.49$ & $1.71 \pm 0.85$ & $<0.001^{*}$ \\
\hline
\end{tabular}

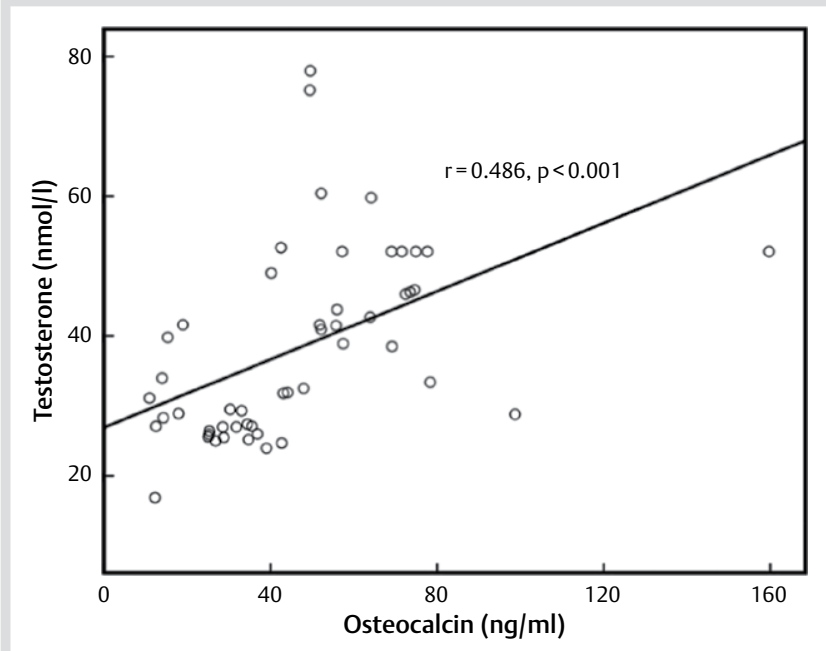

Fig. 1 The association between testosterone and osteocalcin in hyperthyroidism group. In simple Pearson correlation, testosterone was positively associated with Osteocalcin $(r=0.486, P<0.001)$.

(ه Fig. 1), FT3 $(r=0.59, \mathrm{P}=0.000)$ and FT4 $(r=0.575, \mathrm{P}<0.001)$ in the serum ( $\odot$ Table $2 \mathrm{a}$ ), but not related with smoking, drinking, BMI, duration of disease, PTH, CTX, VitD and TSH $(\mathrm{P}<0.05)$. While in the correlation between $\mathrm{OC}$ and respective parameter, OC was also positive related with serum testosterone $(r=0.486$, $\mathrm{P}<0.001)$, FT3 $(\mathrm{r}=0.464, \mathrm{P}=0.003)$ and FT4 $(\mathrm{r}=0.401, \mathrm{P}=0.013)$ and negative related with age $(\mathrm{r}=-0.523, \mathrm{P}=0.001)(\diamond$ Table $2 \mathrm{~b}$ ). The correlation between testosterone and $O C$ was strengthened by the multi-linear regression analysis. OC was positively related with serum testosterone concentration whichever in the model of patients' anthropometric state (Age, BMI, Current smoking, Current drinking and OC involved in Model 1: $R^{2}=0.428$, $\mathrm{P}=0.002$ ), thyroid hormone (Age, BMI, Current smoking, Current drinking, Duration of disease, FT3, FT4 and OC involved in Model
2: $\mathrm{R}^{2}=0.587, \mathrm{P}<0.001$ ), related sex hormones (Age, BMI, Current smoking, Current drinking, Duration of disease, FT3, FT4, FSH, LH and OC involved in Model 3: $\mathrm{R}^{2}=0.409, \mathrm{P}=0.015$ ), or other bone metabolism markers (Age, BMI, Current smoking, Current drinking, Duration of disease, FT3, FT4, FSH, LH, CTX and OC involved in Model 4: $\mathrm{R}^{2}=0.454, \mathrm{P}=0.008$ ) ( $\odot$ Table 3).

\section{Discussion}

$\nabla$

Compared with the control group, the bone resorption marker CTX and the bone formation marker osteocalcin in the serum were significantly higher in the hyperthyroidism group $(\mathrm{p}<0.05)$, which suggested male hyperthyroidism patients in this assay were indeed with high bone turnover. In addition, total testosterone level is higher in the hyperthyroidism group than the control.

In this study, testosterone was negatively correlated with age, and positively related with serum total OC, CTX, FT3 and FT4 in simple Pearson correlation analysis, but in multi-linear regression analysis from model 1 to model 4 , testosterone was only positively correlated with serum total OC. It is reported that testosterone will decline with aging [13], increasing BMI [14] and alcohol use [15] and that smoking will increase testosterone production [16]. We only observed that there is a relationship between testosterone and age in simple Pearson correlation analysis ( $\odot$ Table $2 \mathbf{a}$ ), and that testosterone was only positively correlated with serum total OC after adjusted for age, BMI, smoking, drinking, duration of disease as well as OC in multi-linear regression analysis ( $\odot$ Table 3 - Model 1 ). Some studies revealed that testosterone might relate withFT3 and FT4 in hyperthyroidism patients [17]. We indeed observed it had some relationship with FT3 and FT4, when simple Pearson correlation analysis was performed in our study ( $\odot$ Table $2 \mathbf{a}$ ). To adjust the influence of thyroid hormone itself to testosterone, FT3, FT4 along with age, BMI, smoking, drinking, duration of disease and OC were induced in multi-linear regression analysis, testosterone was only positively correlated with serum total OC ( $\odot$ Table 3 - Model2). In theory, testosterone is regulated by $\mathrm{FSH}$ and $\mathrm{LH}$, so a negative association should be found between testosterone and LH or FSH, whereas such association was not observed in the face of high serum level of osteocalcin in our study (॰ Table 3 - Model3). It is well known that bone metabolism markers contain bone formation marker OC and bone resorption marker CTX. However, the association between serum testosterone and CTX had no statistical significance in simple Pearson correlation analysis ( $\odot$ Table 2a). In addition, when CTX was considered by model 4 in the multiple regression analysis, serum OC instead of CTX had statistical significance.

Taking the above together, we infer in our study that when OC was high, it would play a primary role in mediating testosterone biosynthesis and male reproductive functions regardless of the mediation function of LH, FT3 and FT4 to testosterone. What's more, our findings of significant association between testosterone and osteocalcin level in the face of LH ( $\bullet$ Table 3 - Model3) were consistent with previous report in mice [4] and with some other recent studies [18]. For example, in human, lower osteocalcin level had been shown to be associated with lower testosterone in different energy metabolism diseases $[11,19]$. However, some studies didn't agree with our study. For example, a study in men from infertile couples maintained that osteocalcin was not a strong determinant of serum testosterone and sperm count 


\begin{tabular}{|c|c|c|c|c|c|}
\hline \multirow[t]{2}{*}{ A } & \multicolumn{2}{|c|}{$\mathbf{T}$} & \multirow[t]{2}{*}{ B } & \multicolumn{2}{|c|}{ OC } \\
\hline & $r$ & $\mathbf{P}$ & & $\mathbf{r}$ & $\mathbf{P}$ \\
\hline Age & -0.354 & $0.012^{*}$ & Age & -0.458 & $0.001^{*}$ \\
\hline Current smoking & 0.175 & 0.225 & Current smoking & 0.125 & 0.389 \\
\hline Current drinking & -0.093 & 0.521 & Current drinking & 0.032 & 0.828 \\
\hline Duration & -0.278 & 0.052 & Duration & -0.208 & 0.148 \\
\hline $\mathrm{BMI}$ & -0.057 & 0.728 & $\mathrm{BMI}$ & -0.13 & 0.424 \\
\hline TC & -0.105 & 0.489 & $\mathrm{TC}$ & 0.014 & 0.924 \\
\hline OC & 0.486 & $<0.001^{*}$ & $\mathrm{~T}$ & 0.486 & $<0.001^{*}$ \\
\hline CTX & 0.228 & 0.123 & CTX & 0.285 & 0.052 \\
\hline PTH & -0.241 & 0.120 & PTH & -0.102 & 0.514 \\
\hline 25(OH)VitD & 0.120 & 0.507 & $25(\mathrm{OH})$ VitD & 0.122 & 0.500 \\
\hline FT3 & 0.59 & $<0.001^{*}$ & FT3 & 0.464 & $0.003^{*}$ \\
\hline FT4 & 0.575 & $<0.001^{*}$ & FT4 & 0.401 & $0.013^{*}$ \\
\hline TSH & -0.203 & 0.229 & TSH & -1.141 & 0.262 \\
\hline LH & 0.089 & 0.595 & $\mathrm{LH}$ & -0.026 & 0.877 \\
\hline FSH & -0.273 & 0.108 & FSH & -0.272 & 0.109 \\
\hline
\end{tabular}

Table 2 Simple Pearson correlation between testosterone $(A)$ or total OC (B) and other parameters in the serum in hyperthyroidism group.

Table 3 Multiple linear regression analysis between testosterone and osteocalcin as well as other parameters: Except for osteocalcin, other parameters also involved in different Models were as follow. In the liner regression analysis, testosterone was only positively associated with Osteocalcin in male hyperthyroidism patients and other parameters have no statistical significance in Model 1-4).

\begin{tabular}{|c|c|c|}
\hline & $\mathbf{R}^{2}$ & $\mathbf{P}$ \\
\hline Model1 & 0.428 & 0.002 \\
\hline Model2 & 0.587 & $<0.001$ \\
\hline Model3 & 0.409 & 0.015 \\
\hline Model4 & 0.454 & 0.008 \\
\hline
\end{tabular}

Model 1: Age, BMI, Current smoking, Current drinking and osteocalcin

Model 2: Model 1 + Duration of disease, FT3, FT4, TSH

Model 3: Model2 + FSH, LH

Model 4: Model3 + CTX

[20]. Another study conducted in 614 older male Dutch population (65-88 years) indicated that serum osteocalcin was negatively associated with free and bioavailable testosterone and positively with luteinizing hormone levels [21]. The inconsistency might be related to different ethnic groups and different study population such as the different age, diseases and other reasons. More specifically, the opinions about higher testosterone levels in patients with hyperthyroidism were controversial. Some suggested that the reason was the increased synthesis of sex hormone binding globulin (SHBG) in the liver cells of hyperthyroidism patients [22], which makes the clearance rate of testosterone slow down. Some indicated that the reason was the increased gonadotropin concentration like LH and FSH, for the unreduced function of hypothalamic-pituitary-gonadal axis in patients with hyperthyroidism [23], which was not consistent with our study. In addition, some demonstrated that the reason was the enhanced degradation rate of cholesterol to steroid hormone, which was promoted by thyroid hormone, since steroids could convert into testosterone in adrenal [24]. Lastly, other studies considered the reason was that the thyroid hormone directly acted on the ovary, which increased the secretion of testosterone by ovaries.

Another explanation for the above clinical result was that testosterone affected osteocalcin. Testosterone elevation could promote bone formation and remodeling in senile [25]. Under this process, the OC secretion of osteoblasts would correspondingly increase. In the meantime, as our study was a cross section study, we were not able to exclude that there were other parameters existed that could both influence testosterone and osteocalcin, such as the parameter FT3 and FT4. High testosterone and high total OC were all reported in hyperthyroidism patients. The respective relationship of testosterone or total OC with FT3 or FT4 was indeed observed in our study. However, when total OC, FT3 or FT4 were all considered in the multi-linear regression analysis, we found testosterone has no linear association with FT3 or FT4 in the exsist of total OC, so the third consideration could not also be supported in the population we studied.

The underlying mechanism about how osteocalcin affects testosterone was unclear in human but relatively clear in animal studies. The mechanism was that osteocalcin, in parallel to and independent of the hypothalamus-pituitary-testis axis, via a pancreas-bone-testis axis, promotes testosterone biosynthesis and thus regulates male reproductive functions $[26,27]$. In animal studies, Osteocalcin binds to its receptor Gprc6a on Leydig cells, which favors cAMP production and then induce the activation of the transcription factor CREB (CAMP response element binding). CREB triggers the expression of several genes, likeCyp11a, StAR, 3b-HSD andCyp17, encoding the enzymes that are essential to testosterone biosynthesis [28]. Thus osteocalcin has been increasingly considered to play a critical role in the crosstalk between bone and male fertility. But the undercarboxylated form of osteocalcin (uCOC) might be the main factor acting on Leydig cells as an upstream regulator of reproductive function by favoring testosterone biosynthesis in mice [4], since osteocalcin consisted of both the carboxylated and undercarboxylated form [26] and only ucOC was active in metabolism. However, studies which analyzed this association in human or in male hyperthyroidism patients under the condition of high bone turnover or with high serum level of osteocalcin were limited. Therefore, further prospective studies are needed to verify whether osteocalcin level plays a primary role in the reproductive function of male.

Some limitations still exist in our study. First, the sample size is relatively small. Since some of these discrepancies may due to the underlying differences in individual study population, measurement method and adjustment for potential confounders. Second, serum total osteocalcin is composed of uncarboxylated and carboxylated forms. There have already been animal studies reporting uncarboxylated osteocalcin (ucOC) appears to be the active form and the form of affecting on testosterone $[18,27]$. 
However, it is said total $\mathrm{OC}$ and $\mathrm{ucOC}$ are often with a certain proportion. And several recent clinical studies have demonstrated that not only undercarboxylated but also total osteocalcin were associated with energy metabolism and testosterone as well [11]. Since lack of an automated assay to examine the uncarboxylated form, our study only measured serum total osteocalcin. Thirdly, we'd better introduce sex hormone binding globulin (SHBG) as an adjust parameter for testosterone, for that testosterone in blood composes of free testosterone (1-4\%) and bound testosterone, and the latter loosely combine with to SHBG. In our future study, we will surely measure free testosterone, SHBG and undercarboxylated osteocalcin so as to get a more scientific conclusion. Lastly, we should further emphasize the nature of our cross-sectional study and thus no inferences of causality can be made.

\section{Conclusion}

$\nabla$

In conclusion, the present study suggests that testosterone was positively correlated with serum osteocalcin in male hyperthyroidism patients, which indirectly supported the topic that serum osteocalcin participated in the regulation of sex hormone.

\section{Acknowledgements}

This study was supported by Shanghai Municipal Natural Science Foundation (13ZR1432100) and National High Technology Research and Development Program 863 (2013AA032203).

Disclosure of conflict of interest: The authors declare no conflicts of interest.

\section{References}

1 Kumar TR. Functional analysis of LHß knockout mice. Molecular and cellular endocrinology 2007; 269: 81-84

2 Themmen APNHI. Mutations of Gonadotropins and Gonadotropin Receptors: Elucidating the Physiology and Pathophysiology of Pituitary-Gonadal Function. Endocr Rev 2000; 21: 551-583

3 Ma X, Dong Y, Matzuk MM et al. Targeted disruption of luteinizing hormone-subunit leads to hypogonadism, defects in gonadal steroidogenesis, and infertility. Proceedings of the National Academy of Sciences 2004; 101: 17294-17299

4 Oury F, Ferron M, Huizhen $W$ et al. Osteocalcin regulates murine and human fertility through a pancreas-bone-testis axis. The Journal of clinical investigation 2013; 123: 2421-2433

5 Bolland MJ, Grey A, Horne AM et al. Testosterone levels following decreases in serum osteocalcin. Calcified tissue international 2013; 93: 133-136

$6 \mathrm{Li} Y, \mathrm{Li}$ K. Osteocalcin Induces Growth Hormone/Insulin-Like Growth Factor-1 System by Promoting Testosterone Synthesis in Male Mice. Hormone and metabolic research $=$ Hormon- und Stoffwechselforschung $=$ Hormones et metabolisme 2014

7 Fukumoto S, Martin TJ. Bone as an endocrine organ. Trends in endocrinology and metabolism: TEM 2009; 20: 230-236
8 Karsenty G, Oury F. Biology without walls: The novel endocrinology of bone. Annu Rev Physiol 2012; 74: 87-105

9 Karsenty G, Ferron M. The contribution of bone to whole-organism physiology. Nature 2012; 481: 314-320

10 Hannemann A, Breer S, Wallaschofski $\mathrm{H}$ et al. Osteocalcin is associated with testosterone in the general population and selected patients with bone disorders. Andrology 2013; 1: 469-474

11 Cui $R$, Su B, Sheng $C$ et al. Total osteocalcin in serum predicts testosterone level in male type 2 diabetes mellitus. International journal of clinical and experimental medicine 2014; 7: 1145-1149

$12 \mathrm{Xu} Y$, Wang L, He J et al. Prevalence and control of diabetes in Chinese adults. Jama 2013; 310: 948-959

13 Nardozza Junior A, Szelbracikowski Sdos S, Nardi AC et al. Age-related testosterone decline in a Brazilian cohort of healthy military men. International braz $\mathrm{j}$ urol: official journal of the Brazilian Society of Urology 2011; 37: 591-597

14 Shamim MO, Khan FMA, Arshad R. Association between serum total testosterone and Body Mass Index in middle aged healthy men. Pak J Med Sci 2015; 31: 355-359

15 Maneesh M, Dutta S, Chakrabarti A et al. Alcohol abuse-duration dependent decrease in plasma testosterone and antioxidants in males. Indian journal of physiology and pharmacology 2006; 50: 291-296

16 Wang $W$, Yang $X$, Liang $J$ et al. Cigarette smoking has a positive and independent effect on testosterone levels. Hormones 2013; 12: 567577

17 Ford HC, Cooke RR, Keightley EA et al. Serum levels of free and bound testosterone in hyperthyroidism. Clinical endocrinology 1992; 36 : 187-192

18 Kugelberg E. Reproductive endocrinology: osteocalcin and male fertility. Nature reviews Endocrinology 2013; 9: 441

19 Strapazzon G, Garolla A, Carraro U et al. Osteocalcin and its association with testosterone in patients with metabolic diseases. Osteoporosis international: a journal established as result of cooperation between the European Foundation for Osteoporosis and the National Osteoporosis Foundation of the USA 2013; 24: 2539-2540

20 Schwetz V, Gumpold R, Graupp M et al. Osteocalcin is not a strong determinant of serum testosterone and sperm count in men from infertile couples. Andrology 2013; 1: 590-594

21 Limonard EJ, van Schoor NM, de Jongh RT et al. Osteocalcin and the pituitary-gonadal axis in older men: a population-based study. Clinical endocrinology 2015; 82: 753-759

22 Kostoglou-Athanassiou I, Ntalles K, Gogas $J$ et al. Sex hormones in postmenopausal women with breast cancer on tamoxifen. Hormone research 1997; 47: 116-120

23 Zahringer S, Tomova A, von Werder $K$ et al. The influence of hyperthyroidism on the hypothalamic-pituitary-gonadal axis. Experimental and clinical endocrinology \& diabetes: official journal, German Society of Endocrinology [and] German Diabetes Association 2000; 108: 282-289

24 Skjoldebrand Sparre L, Kollind M, Carlstrom K. Ovarian ultrasound and ovarian and adrenal hormones before and after treatment for hyperthyroidism. Gynecologic and obstetric investigation 2002; 54: 50-55

25 Deb P, Gupta SK, Godbole MM. Effects of short-term testosterone replacement on areal bone mineral density and bone turnover in young hypogonadal males. Indian journal of endocrinology and metabolism 2012; 16: 947-951

26 Ferron M, Lacombe J. Regulation of energy metabolism by the skeleton: Osteocalcin and beyond. Archives of biochemistry and biophysics 2014

27 Overvad S, Bay K, Bojesen A et al. Low INSL3 in Klinefelter syndrome is related to osteocalcin, testosterone treatment and body composition, as well as measures of the hypothalamic-pituitary-gonadal axis. Andrology 2014; 2: 421-427

28 Karsenty G, Oury F. Regulation of male fertility by the bone-derived hormone osteocalcin. Molecular and cellular endocrinology 2014; 382: $521-526$ 